\title{
Mechanism of Protein Tyrosine Phosphatase O Receptor in Hepatocellular Carcinoma
}

\author{
Ye Qin ${ }^{1}$, Xinke Xie ${ }^{2 *}$ \\ ${ }^{1}$ Shaanxi University of Traditional Chinese Medicine, Xianyang 712000, Shaanxi Province, China \\ ${ }^{2}$ The First Affiliated Hospital of Shaanxi University of Traditional Chinese Medicine, Xianyang 712000, Shaanxi Province, \\ China
}

\begin{abstract}
Pathologist Virchow has proposed a hypothesis that the origin of tumors comes from chronic inflammation. Clinically, liver tumors can be divided into three types Hepatocellular carcinoma (HCC) is the most common type, which is closely related to various kinds of inflammation. Studies have shown that protein tyrosine phosphatase receptor type O (PTPRO) is a new type of protein tyrosine phosphatase, which is negatively correlated with tumorigenesis. As a new tumor suppressor protein, PTPRO is of great significance for the diagnosis and treatment of $\mathrm{HCC}$ in the future. This paper aims to discuss the mechanism of PTPRO in HCC.
\end{abstract}

Key words: Protein tyrosine phosphatase type O receptor; Hepatocellular carcinoma; Mechanism

Publication date: March, 2021

Publication online: 31 March, 2021

${ }^{*}$ Corresponding author: Xinke Xie, xiexinke0928 @)163.com

\section{The birth and growth of PTPRO gene}

PTPRO gene, a member of protein tyrosine phosphatase family, belongs to R3 subtype of this family. It was first discovered by Thomas et $\mathrm{al}^{[1]}$ in 1994. At that time, it was found when looking for glomerular specific protein in rabbit model. Therefore, it was named: PTPRO gene Glomerular epithelial protein 1, or GLEPP1, its active protein is mainly distributed in renal glomerular podocytes. The function of this gene in the kidney is to participate in and maintain the structure and function of glomerular cells, and affect the glomerular filtration capacity. In addition, the role of this protein in mice or human vertebrates is highly conserved. The human PTPRO gene is located in $12 \mathrm{p} 13.3-\mathrm{p} 13.2$, which contains 6 transcripts and 1188 amino acids, including 1159 mature proteins. In recent years, the protein receptor is considered to be a tumor suppressor molecule. There are six variants, which exist in human body in full-length form (including liver, kidney, breast, brain, etc.) and truncated form (including B lymphocytes, macrophages). The expression in brain and kidney is relatively higher than that in other tissues. It can inhibit the proliferation of cancer cells and promote the apoptosis of cancer cells. Studies have confirmed that the gene has a great influence on liver cancer, gastric cancer, colorectal cancer, breast cancer, lung cancer and other organs.

\section{PTPRO participates in tumor mechanism through signaling pathway}

\subsection{TLR4、NF-KB}

TOLL like receptor 4 (TLR4) is a common pattern recognition receptor, and it is also the membrane receptor of lipopolysaccharide (LPS). It plays a dual role in the occurrence and development of tumor cells, which can not only promote the tumorigenesis, but also participate in the inhibition ${ }^{[2]}$. The relationship between PTPRO and TLR4 needs to be further studied. Researchers found that there is a signal feedback loop in liver cancer tissue PTPRO, TLR4 and NF- $\kappa \mathrm{B}$ indicate that the feedback regulation and signal transduction between PTPRO and TLR4 are activated by nuclear factor- 
$\kappa \mathrm{B}(\mathrm{NF}-\kappa \mathrm{b})$. Through the detection of NF- $\kappa \mathrm{B}$ inhibition and luciferase reporter gene in 84 cases of human $\mathrm{HCC}$, it was found that the expression of TLR4 and PTPRO were down regulated, while the expression of TLR4 was significantly up-regulated in PTPRO overexpressed HCC cells. The decreased expression of TLR4 and the inhibition of TLR4/NF$\kappa \mathrm{B}$ signal transduction in $\mathrm{HCC}$ cells may be related to tumor immune tolerance and immune escape in liver immune microenvironment. Stimulation of hepatoma cells with LPS can enhance TLR4 signaling pathway and NF- $\kappa B$ phosphorylation. Meanwhile, the expression of PTPRO gene increases correspondingly. PTPRO promoter lacking NF $-\kappa \mathrm{B}$ binding site can not be activated by LPS, indicating that this feedback regulation of PTPRO and TLR4 in HCC may be realized through NF- $\mathrm{KB}$ activation $^{[3]}$. Other studies have found that PTPRO in hepatocytes can alleviate liver injury, on the contrary, PTPRO in macrophages can aggravate liver injury, which indicates that PTPRO in different immune microenvironment may have different regulatory roles, and this role is also achieved through feedback regulation of its NF- $\kappa \mathrm{B}$ activity ${ }^{[4]}$. It is found that the expression of PTPRO and TLR4 in some human specimens with HCC is significantly lower than that in paracancerous tissues, and there is a significant positive correlation between them. Preliminary experiments show that PTPRO may be up-regulated in the inflammatory microenvironment and play a role in the regulation of inflammation. Therefore, under the stimulation of inflammation, the protein receptor shows a high expression and regulates the progress of inflammatory response, leading to inflammation The role of neurosis in tumor can not only promote its occurrence and development, growth and infiltration, but also inhibit its decline ${ }^{[5]}$. Researchers have not conducted animal experiments in vivo and in vitro on this signaling pathway, so it can be further studied.

\subsection{Estrogen receptor alpha}

Today, the incidence rate of HCC is much higher than that of women. The research on this apparent gender difference has begun. It is found that estrogen receptor alpha (ER) plays an important role in the development of HCC. The mechanism has been partially elucidated, such as inhibiting the growth of by inhibiting hepatocyte growth factor, interleukin
6, and anti-inflammation effects ${ }^{[6]}$. Some studies have found that women's risk of HCC is inversely proportional to menopausal age and the number of full-term pregnancies, and in animal studies, ovariectomy increases the susceptibility of female mice to liver cancer. In the clinical study of patients with liver cancer, it was found that there was gender difference in the expression level of PTPRO in paracancerous tissues, which was significantly lower in male patients than in female patients. This gender difference was caused by the regulation of Er $\alpha$, indicating that estrogen and its receptor (ER) were involved in the occurrence and development of HCC. It was found that ER $\alpha$ could up regulate the expression of PTPRO as a transcription factor ${ }^{[7]}$. There is estrogen estrogen receptor complex in breast cancer, which can regulate the transcription of PTPRO. In the process of regulation, er $\alpha$ and ER $\beta$ play opposite roles. The main role in breast cancer is the inhibition of $\operatorname{Er} \beta$ on PTPRO, thus inhibiting the occurrence and development of tumor ${ }^{[8]}$. In the study of HCC, it was found that ER $\alpha$ level was significantly decreased in tumor area, but there was no significant difference in ER $\beta$ level. Therefore, the role of $\mathrm{Er} \alpha$ in hepatocellular carcinoma was paid special attention. In addition, estrogen can inhibit liver cancer cells, reduce the activity of NF$\kappa \mathrm{B}$ signaling pathway, regulate downstream related genes, reduce the progress of inflammatory response, affect the proliferation and migration of cancer cells, and induce apoptosis of cancer cells ${ }^{[9]}$.

\section{Conclusion}

As the pathogenesis of HCC is complex, the early diagnosis is hidden, the postoperative metastasis and recurrence are easy, and the late stage is difficult to treat. The average 3 -year survival rate is about $13 \%$ $21 \%$, which involves many factors. Therefore, it is of great significance to explore the mechanism and find a better targeted treatment plan ${ }^{[10]}$. PTPRO plays an anti-tumor role in human tumors and cancer cells, and inhibits tumorigenesis through multiple signaling pathways. At present, our research on the mechanism of PTPRO gene in tumor is in the initial stage. Further research will help us to understand the pathogenesis of liver cancer and find potential therapeutic targets for the disease. 


\section{References}

[1] Zhang DY, Liu FR. Research progress of PTPRO in digestive system tumors $[\mathrm{J}]$. Journal of clinical and Experimental Pathology, 2015, 31(8): 908-911.

[2] Jiang BR, Chu YJ, Zhang DK. The role of Toll like receptor 4 in the development of hepatocellular carcinoma [J]. Journal of Clinical Gastroenterology, 2020, 32 (1): 61-65.

[3] Xu D, Wang X, Yan S, et al. Interaction of PTPRO and TLR4 signaling in hepatocellular carcinoma[J]. Tumor Biology, 2014, 35(10): 10267-10273.

[4] Wu L, Huang T, Chen HH. Research on PTPRO in liver diseases [J]. Chinese Journal of Modern Medicine, 2016,18 (10): 98-101.

[5] Luo XX, Chen GD, Jiao Y, et al. Research status of TLR4 in inflammatory cancer [J]. Chinese Journal of Immunology, 2017,33 (11): 1735-1740.

[6] Lian JY, Tuo BG, Wen GR, et al. Research progress of estrogen receptor in digestive system tumor [J]. World Journal of Chinese digestion, 2015, 23(26): 4227-4235.
[7] Hou JJ, Jiang RQ, Deng L, et al. Gender difference of PTPRO expression in hepatocellular carcinoma and its relationship with prognosis [J]. Journal of Nanjing Medical University (NATURAL SCIENCE EDITION), 2012, 32 (6): 784-789.

[8] Ren W, Wang X, Bao Y, et al. Estrogen inhibits receptor tyrosine phosphatase $\mathrm{O}$ and promotes proliferation of mouse podocytes [J]. Journal of Molecular Imaging, 2018, 41(4): 520-524.

[9] Li YM, Jia SY, Xu AJ, et al. Effect of estrogen on oncological behavior of hepatoma cells through NF - $\kappa$ B signaling pathway [J]. Journal of Clinical and Experimental Medicine, 2019, 18(2): 9-14.

[10] Zhang SN, Guo XJ, Chen G. Correlation analysis of differential methylation sites in prognosis of hepatocellular carcinoma [J]. Journal of Hepatobiliary and Pancreatic Surg, 2019, 31(1): 47-53.

[11] Zhang SN, Guo XJ, Chen G. Correlation analysis of differential methylation sites in prognosis of hepatocellular carcinoma [J]. Journal of Hepatobiliary and Pancreatic Surg, 2019, 31(1): 47-53. 